\title{
Perfil epidemiológico das hepatites B e C em Maceió-AL no período de 2010-2020
}

\author{
Epidemiological profile of hepatitis B and C in Maceió-AL in the period of 2010-2020 \\ Perfil epidemiológico de hepatitis B y C en Maceió-AL en el periodo 2010-2020
}

Dinah Lopes Marques Luz ${ }^{1 *}$, Bruna Letícia Gomes Costa Wanderley¹, Antonio Fernando Silva Xavier Júnior², Laércio Pol-Fachin¹.

\section{RESUMO}

Objetivo: Caracterizar o perfil epidemiológico dos pacientes infectados pelo vírus da hepatite $\mathrm{B}$ e $\mathrm{C}$ no período de 2010-2020 na cidade de Maceió - AL. Métodos: Trata-se de um estudo observacional, descritivo, do tipo transversal, realizado a partir de dados não nominais, extraídos do Sistema de Informação de Agravos de Notificação (SINAN), disponibilizados em planilhas de Excel pela secretaria de saúde municipal. A análise dos dados foi feita por meio de estatística descritiva das variáveis estudadas: sexo, faixa etária, raça/cor, nível de escolaridade e apresentação clínica. As diferenças foram consideradas significativas para os valores de $p$ menores que 0,05. Resultados: O número de infectados por hepatites virais no município de Maceió de 2010 a 2020 foi de 2.570 casos. As infecções mais prevalentes foram decorrentes de HBV e HCV, sem qualquer notificação de HDV e HEV. Conclusão: Na capital alagoana as hepatites mais predominantes são B e C, onde a B foi a mais prevalente de 2010 a 2020. Sendo o sexo masculino, indivíduos na faixa etária dos 40 aos 59 anos, de raça parda, com ensino fundamental incompleto e em fase crônica da doença os mais acometidos.

Palavras-chave: Hepatites virais, Hepatite B, Hepatite C.

\begin{abstract}
Objective: To characterize the epidemiological profile of patients infected by the hepatitis $B$ and $C$ virus in the period 2010-2020 in the city of Maceió - AL. Methods: This is an observational, descriptive, cross-sectional study, carried out from non-nominal data, extracted from the Notifiable Diseases Information System (SINAN), available in Excel spreadsheets by the municipal health department. Data analysis was performed using descriptive statistics of the studied variables: gender, age group, race/color, education level and clinical presentation. Differences were considered significant for $p$ values less than 0.05 . Results: The number of infected with viral hepatitis in the city of Maceió from 2010 to 2020 was 2,570 cases. The most prevalent infections were caused by HBV and HCV, without any notification of HDV and HEV. Conclusion: In the capital of Alagoas, the most prevalent hepatitis are B and C, where B was the most prevalent from 2010 to 2020 . The sex, belonging to the age group of 40 to 59 years, of mixed race, with incomplete elementary education and in the chronic phase of the disease the most affected.
\end{abstract}

Keywords: Viral hepatitis, Hepatites B, Hepatites C.

\section{RESUMEN}

Objetivo: Caracterizar el perfil epidemiológico de los pacientes infectados por el virus de la hepatitis $\mathrm{B}$ y $\mathrm{C}$ en el período 2010-2020 en la ciudad de Maceió - AL. Métodos: Se trata de un estudio observacional, descriptivo, transversal, realizado a partir de datos no nominales, extraídos del Sistema de Información de Enfermedades

\footnotetext{
${ }^{1}$ Centro Universitário CESMAC, Maceió - AL. *E-mail: dinahmluz@gmail.com

${ }^{2}$ Secretaria Municipal de Saúde, Maceió - AL.
}

SUBMETIDO EM: 11/2021 
Notificables (SINAN), disponible en hojas de cálculo Excel por el departamento de salud municipal. El análisis de los datos se realizó mediante estadística descriptiva de las variables estudiadas: sexo, grupo de edad, raza / color, nivel educativo y presentación clínica. Las diferencias se consideraron significativas para valores de $p$ inferiores a 0,05. Resultados: El número de infectados por hepatitis virales en la ciudad de Maceió de 2010 a 2020 fue de 2.570 casos. Las infecciones más prevalentes fueron por VHB y VHC, sin notificación de VHD y VHE. Conclusión: En la capital de Alagoas, las hepatitis más prevalentes son $B$ y $C$, donde $B$ fue la más prevalente de 2010 a 2020. El sexo, perteneciente al grupo de edad de 40 a 59 años, mestizo, con educación primaria incompleta y en la fase crónica de la enfermedad es la más afectada.

Palabras clave: Hepatites virais, Hepatitis B, Hepatitis C.

\section{INTRODUÇÃO}

As Hepatites Virais (HV), são doenças de notificação compulsória, e um importante problema de saúde pública global que afeta milhões de pessoas, estando associadas a uma morbidade e mortalidade significativas, com altos custos de tratamento e também aumento no do número de transplantes hepático (LANINI S, et al., 2019; SILVA TGQ, et al.,2020).Tais doenças apresentam um processo inflamatório caracterizado pela lesão dos hepatócitos e recrutamento de células inflamatórias, podendo evoluir de forma aguda ou crônica, de forma assintomática ou sintomática, a depender do agente viral envolvido e da resposta imunológica do paciente (EVANGELISTA CBA, et al., 2021; NUNES HM, et al., 2017; SILVA TG, et al.,2020). Quanto ao diagnóstico nota-se que geralmente é realizado tardiamente, pois, na grande maioria dos casos a doença apresenta-se oligossintomática ou até mesmo assintomática (VIANA DR, et al.,2017).

Atualmente, são conhecidos cinco vírus responsáveis pelas diferentes hepatites humanas: os vírus das hepatites A (HAV), B (HBV), C (HCV), D (HDV) e E (HEV) (NUNES HM, et al., 2017). As hepatites virais B e $C$ afetam 325 milhões de pessoas no mundo, ocasionando 1,4 milhão mortes por ano, perdendo apenas para tuberculose dentre as causas de morte por doenças infecciosas (MINSTÉRIO DA SAÚDE, 2019).

Ao longo dos vinte anos compreendidos entre 1999 e 2019, foram notificados, no Brasil, 673.389 de casos confirmados de hepatites virais no Sistema de Informação de Agravos de Notificação (SINAN). De acordo com esses dados, a maioria dos casos são referentes à hepatite $C(253.307,37,6 \%)$, seguidos de casos de hepatite B $(247.890,36,8 \%)$, hepatite A $(168.036,25,0 \%)$ e hepatite D $(4.156,0,6 \%)$ (MINSTÉRIO DA SAÚDE, 2020).

Observou-se uma variação na distribuição dos casos entre as cinco regiões brasileiras. As infecções pelo vírus A têm maior proporção na região do Nordeste (30,1\%). A região Sudeste apresentou as maiores concentrações do HBV e HBC, com $34,5 \%$ e $59,3 \%$, respectivamente. Enquanto na região Norte acumula $74,4 \%$ do total de casos de HDV. Segundo os dados disponibilizados pelo DATASUS, entre o período de 1999 e 2019, houve maior prevalência de hepatite B em Maceió-AL (MINSTÉRIO DA SAÚDE, 2020).

A infecção causada pelo vírus B possui um período de incubação que varia de 30 a 180 dias, tendo como principais vias de transmissão: perinatal, parceiros sexuais e por via parenteral (EVANGELISTA CBA, et al.,2021). Pode apresentar uma variedade de apresentações clínicas, desde portador assintomático a manifestações graves como hepatite crônica, hepatite fulminante, cirrose e Carcinoma Hepatocelular (CHC) (OLIVEIRA MS, et al., 2019).

A forma crônica da doença, quando o vírus permanece por mais de seis meses, é observada entre $5 \%$ a $10 \%$ dos adultos infectados pelo HBV, sendo responsável pela terceira e quarta posição de causas de cirrose (MINSTÉRIO DA SAÚDE, 2018). Entretanto, geralmente a doença evolui com bom prognóstico, onde o indivíduo fica livre do vírus em cerca de $90 \%$ a $95 \%$ dos casos (LANINI S, et al., 2019).

Com relação à história natural da doença da hepatite $C$, o período de incubação da infecção do vírus do HCV é de 14 a 180 dias e menos de $15 \%$ das pessoas infectadas desenvolvem doenças agudas (LANINI S, et al., 2019). Sua transmissão ocorre através do sangue por vias de compartilhamento de objetos perfuro cortantes como: injeções, alicate ou por via sexual ou transfusão sanguínea (LEÃO DV, et al., 2021). Até o 
início da década de 90, a principal forma de transmissão era por transfusão sanguínea. Contudo, com a implantação da triagem sorológica para anti-HCV em bancos de sangue de verias nações, houve uma redução importante desse meio de infecção (SANTOS ECC E MEMORIAN MAP, 2021). A cronicidade da doença causada por HCV varia entre 60 e 90\%, sendo maior de acordo com algumas características do indivíduo, incluindo ser do sexo masculino, idade maior que 40 anos, coinfecções e comorbidades como hepatite B, esquistossomose, HIV, alcoolistas, obesidade e resistência à insulina (LANINI S, et al., 2019).

A coinfecção HCV e o Vírus da Imunodeficiência Humana (HIV) é recorrente e cerca de $70 \%$ a $90 \%$ dos pacientes soropositivos para o HIV já tiveram contato com as hepatites virais. Na Europa e nos Estados Unidos, cerca de 1/3 dos pacientes portadores do HIV estão coinfectados com HCV. Esta simultaneidade entre as infecções é um fator complicador da doença pois há maior chance de evoluir para forma grave (FERREIRA VL, et al., 2017; NUNES FS, et al., 2020). Dentre elas, o desenvolvimento de carcinoma hepatocelular (DAS M, 2021).

O diagnóstico das hepatites virais é realizado a partir da avaliação clínica, associado a exames laboratoriais inespecíficos, dentre eles prova de função, lesão hepática e sorologias específicas. Na avaliação clínica pode-se observar colúria, mal-estar, náuseas, vômitos. Contudo, a doença tende a evoluir de forma silenciosa ou com sintomas inespecíficos, dificultando tal suspeita clínica e consequentemente a não investigação laboratorial (MINSTÉRIO DA SAÚDE, 2019; NUNES FS, et al., 2020). Diante disso, a grande maioria das pessoas infectadas desconhecem a doença e o HCV pode ser considerado uma das principais hepatopatias crônica do Brasil (MINSTÉRIO DA SAÚDE, 2017). Um estudo realizado nos Estados Unidos observou que pelo menos metade dos adultos infectados pelo vírus $C$ não têm conhecimento do seu diagnóstico (UDOMPAP P, et al.,2016).

Frente a este problema de saúde pública, na década de 90 , as vacinas contra os vírus $\mathrm{A}$ e $\mathrm{B}$, entraram do Programa Nacional de Imunização (PNI), sendo que inicialmente a prevenção era restrita aos grupos de risco (INTERFARMA, 2016; GUSMÃO BM, et al., 2017). A vacina contra o HAV, consiste em duas doses com intervalo de 6 meses cada, sendo recomendação da sociedade brasileira de pediatria que seja aplicada aos 12 e 18 meses de vida. Já a vacina contra o HBV, consiste em quatro doses, sendo a primeira aplicada nas primeiras 12-24h de vida, aos 2, 4 e 6 meses de vida. Quando completar o esquema vacinal o ideal é que sejam feitos testes para confirmar se houve desenvolvimento de anticorpos contra o HBV (anti-HBS) (GUSMÃO BM, et al., 2017). Ainda como uma forma de promoção de saúde e prevenção de doença, foi criada, a lei $n^{\circ} 13.802$ de 10/01/2019 que institui o julho Amarelo, a ser realizado a cada ano, em todo o território nacional. No mês de julho. Portanto, são realizadas ações relacionadas à luta contra as hepatites virais. Uma forma de conscientizar a população sobre tais doenças (BRASIL, 2019).

Diante do exposto, nota-se que há uma maior prevalência de casos de hepatite $B$ e $C$ no Brasil. Observase, também, uma maior probabilidade, principalmente do vírus $C$, da cronificação da doença, tornando o diagnóstico precoce fundamental. Diante disso, é imprescindível descrever o perfil epidemiológico dos pacientes infectados pelo HCV E HBV para traçar estratégias direcionadas de promoção de saúde e prevenção da doença. Desta forma, o objetivo do presente artigo foi descrever o perfil epidemiológico dos pacientes infectados pelo vírus da hepatite B e C no período de 2010-2020 na cidade de Maceió - AL.

\section{MÉTODOS}

Trata-se de um estudo observacional, descritivo, do tipo transversal, realizado a partir de dados não nominais, extraídos do Sistema de Informação de Agravos de Notificação (SINAN), disponibilizados em planilhas de Excel (Microsoft Office ${ }^{\circledR}$ ) pela secretaria de saúde municipal de Maceió - AL. A amostra foi composta por 1.398 indivíduos diagnosticados e notificados com hepatite B e/ou C em Maceió - AL entre os anos de 2010 e 2020. Foram observadas as variáveis demográficas: sexo, faixa etária, raça/cor, nível de escolaridade e apresentação clínica. Foram excluídos qualquer tipo de hepatite que não $B$ e $C$ notificados no município de Maceió entre o período pesquisado.

A análise dos dados foi feita por meio de estatística descritiva, através do cálculo de frequências absolutas e relativas para as variáveis estudadas, e organizadas em tabelas no software Microsoft Excel®. As 
frequências obtidas foram posteriormente analisadas por estatística analítica, através da aplicação do teste qui-quadrado $\left(\square^{2}\right)$ para comparação de proporções, e dos testes de normalidade de Shapiro-Wilk e de MannWhitney para comparação de grupos. As diferenças foram consideradas significativas para os valores de $p$ menores que 0,05 .

Considerando que os dados coletados estão disponíveis em banco de acesso público, não houve necessidade de submissão a um Comitê de Ética em Pesquisa com seres humanos, em conformidade com a Resolução no 510/2016 do Comitê Nacional em Saúde.

\section{RESULTADOS E DISCUSSÃO}

O número de infectados por hepatites virais no município de Maceió de 2010 a 2020 foi de 2.570 casos, dentre esses $801(31,17 \%)$ foram registrados como ignorados/brancos para o tipo de hepatite viral. Em relação aos demais, observou-se que as infecções mais prevalentes foram decorrentes de HBV e HCV, sem qualquer notificação de HDV e HEV (Tabela 1). Não se observou diferença estatisticamente significativa entre a frequência de ocorrência de hepatites $B$ e $C(\square 2, p=0.379)$, nem entre o quantitativo de casos, ao longo dos anos avaliados (teste de Mann-Whitney, $p=0.599$ ). Por outro lado, ambas as hepatites $B$ e $C$ tem percentual estatisticamente maior de casos $\left(\square^{2}, p<0.0001\right)$ do que qualquer outra hepatite (grupo "Outros"), bem como quantitativo de casos maior (teste de Mann-Whitney, $p=0.020$, HBV versus "Outros"; $p=0.039$, HCV versus "Outros").

A maior prevalência das hepatites supracitadas corrobora com os dados encontrados no boletim epidemiológico viral de 2020, que sinaliza que as hepatites mais prevalentes no Brasil entre 1999 e 2019 foram a B e C (MINSTÉRIO DA SAÚDE, 2020). Contudo, o estudo afirma que a mais prevalente no Nordeste é a tipo $A$, o que não observamos na capital alagoana.

Tabela 1 - Distribuição de frequência (absoluta e relativa) de pessoas portadoras de Hepatite notificada entre o ano de 2010-2020.

\begin{tabular}{lcc}
\hline Hepatite & Quantidade de casos & Percentual \\
\hline Ignorado & 801 & $31,17 \%$ \\
Vírus B & 713 & $27,74 \%$ \\
Vírus C & 685 & $26,65 \%$ \\
Vírus B+C & 22 & $0,87 \%$ \\
Outros & 349 & $13,57 \%$ \\
TOTAL & 2570 & $100 \%$ \\
\hline
\end{tabular}

Legendas: Outros $=$ Vírus $A$, vírus $A+B$ e vírus $A+C$. Fonte: Luz DLM, et al.,2021; dados extraídos do SINAN.

Conforme o sexo, observou-se que o masculino foi o mais predominante em ambas HV. No caso de HBV a prevalência foi de $51,61 \%$ em homens, contra $48,11 \%$ de casos em mulheres, demonstrando uma relação entre os sexos de 1,07 vezes maior de casos masculino em comparação ao feminino, sem significância estatística $\left(\chi^{2}, p=0.186\right)$. Em se tratando de HCV, $56,93 \%$ dos casos ocorreram em indivíduos do sexo masculino e $42,93 \%$ do feminino, o que reflete uma proporção 1,32 vezes maior no homem que na mulher, e que há estatisticamente mais homens infectados pelo HCV do que mulheres $\left(\chi^{2}, p<0.0001\right)$. Corroborando com o encontrado em outros estudos onde os homens, também, foram os mais acometidos (Tabela 2) (ARAÚJO IN, et al., 2020; BARBOSA GFD, et al., 2019; GOMES AM, et al., 2020; OLIVEIRA TJB, et al., 2018; SOTERO AS, et al., 2020).

Tabela 2 - Distribuição de frequência (absoluta e relativa) de pessoas portadoras de Hepatite B e C, segundo sexo, notificada entre o ano de 2010-2020.

\begin{tabular}{lcc}
\hline \multirow{2}{*}{ Sexo } & \multicolumn{2}{c}{ Quantidade de casos (percentual) } \\
\cline { 2 - 3 } & Hepatite B & Hepatite C \\
\hline Ignorado & $2(0,28 \%)$ & $1(0,14 \%)$ \\
Feminino & $343(48,11 \%)$ & $294(42,93 \%)$ \\
Masculino & $368(51,61 \%)$ & $390(56,93 \%)$ \\
TOTAL & $713(100 \%)$ & $685(100 \%)$ \\
\hline
\end{tabular}

Fonte: Luz DLM, et al.,2021; dados extraídos do SINAN. 
Stockdale AJ, et al. (2020) observaram que a prevalência do vírus B foi maior em usuários de drogas injetáveis, usuários de hemodiálise, homens que fazem sexo com homens, profissionais do sexo e aqueles com HCV ou HIV. Pereira VRZB, et al. (2017) também mencionam que ter um membro da família infectado com hepatite B estar fortemente associado a infecção por esse vírus. Segundo dados do Ministério da Saúde, a maioria das infecções pelo HBV ocorreram por via sexual, totalizando $21,3 \%$ dos casos nos últimos 20 anos (MINSTÉRIO DA SAÚDE, 2020).

Já Santos ECC e Memorian MAP (2021), observaram a situação epidemiológica brasileira sobre as hepatites B e C no período de 2000 a 2016, constataram uma dificuldade para analisar a provável fonte/mecanismo de infecção, devido a uma grande restrição na análise dessa variável, pelo fato de mais de $60 \%$ desses dados serem ignorados ou deixados em branco. Contudo, quando tais casos eram excluídos a distribuição dos casos de hepatite B tinham a via sexual como a forma predominante de transmissão.

A faixa etária mais prevalente, considerando ambas as hepatites, foi a dos indivíduos entre 40 e 59 anos, o que representa $42,91 \%$ desses portadores (600/1398). Quanto ao HCV, observa-se uma maior prevalência na faixa etária supracitada, seguida dos idosos entre 60 e 69 anos. Por outro lado, nos casos de HBV, notase que a maior prevalência foi entre jovens de 20 e 39 anos, seguidos dos adultos entre 40 e 59 anos (Tabela 3). Dados também observados em Leão DV, et al. (2021) no estudo realizado na região norte do país.

Tabela 3 - Distribuição de frequência (absoluta e relativa) de pessoas portadoras de Hepatite B e C, segundo faixa etária.

\begin{tabular}{lccc}
\hline \multirow{2}{*}{ Faixa etária } & \multicolumn{2}{c}{ Quantidade de casos (percentual) } & \multirow{2}{*}{ p-valor $^{\text {a }}$} \\
\cline { 2 - 3 } & Hepatite B & Hepatite C & \\
\hline$<20$ anos & $23(3,23 \%)$ & $12(1,75 \%)$ & 0.077 \\
20 a 39 anos & $300(42.08 \%)$ & $85(12,4 \%)$ & $<0.0001$ \\
40 a 59 anos & $294(41,23 \%)$ & $306(44,67 \%)$ & 0.194 \\
60 a 69 anos & $79(11,08 \%)$ & $211(30,8 \%)$ & $<0.0001$ \\
$>70$ anos & $17(2,38 \%)$ & $71(10,36 \%)$ & $<0.0001$ \\
TOTAL & $713(100 \%)$ & $685(100 \%)$ & - \\
\hline
\end{tabular}

Fonte: Luz DLM, et al.,2021; dados extraídos do SINAN.

No presente estudo pode-se observar um percentual estatisticamente maior de infectados pelo HBV, em comparação com HCV, na faixa etária dos 20 aos 39 anos $\left(\chi^{2}, p<0.0001\right)$, bem como um percentual estatisticamente maior de infectados pelo HCV, em comparação com HBV, em idosos $\left(\chi^{2}, p<0.0001\right)$, tanto na faixa etária dos 60 aos 69 anos, quanto na faixa etária dos maiores de 70 anos.

Já conforme a raça, nota-se que $62,94 \%$ dos casos (880/1398) são de pessoas pardas (Tabela 4). Para ambas as hepatites $\mathrm{B}$ e C, observa-se uma distribuição em ordem decrescente, com uma maior prevalência de pardos, seguidos de brancos e pretos. Ao se comparar a prevalência das diferentes etnias entre as duas infecções, pode-se observar um percentual estatisticamente maior de infectados brancos pelo HCV, em comparação com HBV $\left(\chi^{2}, p=0.030\right)$.

Tabela 4 - Distribuição de frequência (absoluta e relativa) de pessoas portadoras de Hepatite B e C, segundo a raça.

\begin{tabular}{|c|c|c|c|}
\hline \multirow{2}{*}{ Raça } & \multicolumn{2}{|c|}{ Quantidade de casos (percentual) } & \multirow{2}{*}{$\mathrm{p}$-valor ${ }^{\mathrm{a}}$} \\
\hline & Hepatite B & Hepatite C & \\
\hline Ignorado & $136(19,07 \%)$ & $143(20,87 \%)$ & 0.400 \\
\hline Branca & $55(7,71 \%)$ & $76(11,09 \%)$ & 0.030 \\
\hline Preta & $52(7,29 \%)$ & $34(4,96 \%)$ & 0.069 \\
\hline Amarela & $11(1,55 \%)$ & $9(1,31 \%)$ & 0.706 \\
\hline Indígena & $1(0,14 \%)$ & $1(0,14 \%)$ & 1.000 \\
\hline Parda & $458(64,24 \%)$ & $422(61,06 \%)$ & 0.219 \\
\hline Total & $713(100 \%)$ & $685(100 \%)$ & - \\
\hline
\end{tabular}

Fonte: Luz DLM, et al.,2021; dados extraídos do SINAN. 
Segundo o Ministério da Saúde, 2020, a distribuição proporcional dos casos segundo raça/cor, em 2019, mostrou pela primeira vez uma alteração, com a maior concentração entre as pessoas autodeclaradas pardas e pretas, totalizando $55,1 \%$ sendo $44,1 \%$ de pardas e $11 \%$ pretas, seguidas das brancas com $42,5 \%$ (BRASIL, 2020).

Quanto a escolaridade os casos de pessoas com ensino fundamental incompleto foram os mais prevalentes com 26,82\% (375/1398), seguido de 17,38\% (243/1398) de indivíduos com ensino médio completo. Ao analisar as hepatites B e C isoladamente, também se nota uma maior prevalência das escolaridades já mencionadas (Tabela 5). Já Leite JMS, et al. (2019) observaram em seu estudo com pacientes com HCV, que as escolaridades mais prevalentes foram de nível de ensino fundamental incompleto e médio incompleto. A maior proporção de infectados em indivíduos com níveis de escolaridade mais baixo, pode ter relação com as características de âmbito social, uma vez que a porcentagem da população com ensino superior completo ou incompleto é bem inferior aos dos demais (MINSTÉRIO DA SAÚDE, 2018).

Tabela 5 - Distribuição de frequência (absoluta e relativa) de pessoas portadoras de Hepatite B e C, segundo a escolaridade.

\begin{tabular}{lccc}
\hline \multirow{2}{*}{ Escolaridade } & \multicolumn{2}{c}{ Quantidade de casos (percentual) } & \multirow{2}{*}{ p-valor } \\
\cline { 2 - 3 } & Hepatite B & Hepatite C & \\
\hline Ignorado & $234(32,82 \%)$ & $236(34,45 \%)$ & 0.519 \\
Analfabeto & $26(3,65 \%)$ & $40(5,84 \%)$ & 0.053 \\
EF incompleto & $213(29,87 \%)$ & $162(23,65 \%)$ & 0.008 \\
EF completo & $28(3,93 \%)$ & $34(4,96 \%)$ & 0.350 \\
EM incompleto & $46(6,45 \%)$ & $37(5,40 \%)$ & 0.406 \\
EM completo & $117(16,41 \%)$ & $126(18,39 \%)$ & 0.329 \\
ES incompleta & $22(3,08 \%)$ & $8(1,17 \%)$ & 0.013 \\
ES completa & $27(3,79 \%)$ & $42(6,13 \%)$ & 0.043 \\
TOTAL & $713(100 \%)$ & $685(100 \%)$ & - \\
\hline
\end{tabular}

Legendas: $\mathrm{EF}=$ ensino fundamental; $\mathrm{EM}=$ ensino médio; $\mathrm{ES}=$ ensino superior. ${ }^{\mathrm{a}} \chi^{2}$ para comparação de proporções. Fonte: Luz DLM, et al.,2021; dados extraídos do SINAN.

Ao comparar-se a prevalência das diferentes escolaridades entre as duas infecções, pode-se observar um percentual estatisticamente maior de infectados analfabetos e com ensino superior incompleto pelo HBV, em comparação com HCV $\left(\chi^{2}, p=0.008\right.$ e 0.013 , respectivamente). Adicionalmente, ao analisar a distribuição dos casos nos indivíduos com ensino superior completo, a prevalência do vírus $C$ é quase o dobro do vírus $B$, o que reflete em uma diferença estatisticamente significativa $\left(\chi^{2}, p=0.043\right)$.

O diagnóstico das hepatites virais é um desafio, devido a prevalência da forma assintomática. Tal diagnóstico é feito a partir da avaliação clínica, exames laboratoriais inespecíficos, prova de função hepática e sorologias específicas. Sinais e sintomas como mal-estar, náuseas, vômitos, icterícia, colúria, fezes de cor esbranquiçada, fadiga e outros podem apresentar-se nesses pacientes. Dentre os exames laboratoriais inespecíficos dosa-se aminotransferases, que indica lesão do parênquima hepático, com aumento do até 100 vezes acima do normal na forma aguda da doença. Para prova de função hepática podem ser dosados proteínas séricas, atividade de protrombina, fosfatase alcalina, dentre outros. Já quanto as sorologias, para HBV pode ser solicitado HbsAg, anti-HBS, anti-HBC IgM e IgG, já para hepatite C solicita-se anti-HCV (MINSTÉRIO DA SAÚDE, 2019; NUNES FS, et al., 2020).

No presente estudo, à forma clínica mais prevalente em ambos os vírus $\mathrm{B}$ e $\mathrm{C}$, no momento da notificação, foi hepatite crônica/portador assintomático, em mais de $70 \%$ dos casos. As formas agudas ocorreram em $12,48 \%$ e 7,29\%, respectivamente (Tabela 6). Observou-se diferença estatisticamente significativa somente entre portadores com hepatite aguda, com percentual maior entre portadores de HBV $\left(\chi^{2}, p=0.001\right)$. Segundo Nunes FS, et al. (2020) o diagnóstico ocorre, em sua maioria, na fase crônica da doença principalmente nos casos do vírus $\mathrm{B}$ e $\mathrm{C}$. 
Tabela 6 - Distribuição de frequência (absoluta e relativa) de pessoas portadoras de Hepatite B e C, segundo a apresentação clínica da doença.

\begin{tabular}{|c|c|c|c|c|}
\hline \multirow{2}{*}{ Apresentação Clínica } & & \multicolumn{2}{|c|}{ Quantidade de casos (percentual) } & \multirow{2}{*}{$p$-valor ${ }^{a}$} \\
\hline & & Hepatite B & Hepatite C & \\
\hline Ignorado & & $47(6,60 \%)$ & $56(8,17 \%)$ & 0.261 \\
\hline $\begin{array}{ll}\text { Hepatite } & \text { Crônica/ } \\
\text { assintomático } & \end{array}$ & portador & $536(75,17 \%)$ & $534(77,95 \%)$ & 0.220 \\
\hline Inconclusivo & & $41(5,75 \%)$ & $45(6,56 \%)$ & 0.528 \\
\hline Hepatite Aguda & & $89(12,48 \%)$ & $50(7,29 \%)$ & 0.001 \\
\hline Total & & $713(100 \%)$ & $685(100 \%)$ & - \\
\hline
\end{tabular}

Fonte: Luz DLM, et al.,2021; dados extraídos do SINAN.

No Brasil $77 \%$ dos casos de hepatites são diagnosticadas na forma crônica seguida de hepatite aguda com $13 \%$, esse perfil está correlacionado à manifestação clínica assintomática da doença, que faz o doente só procure os serviços de saúde quando iniciar os sintomas da fase crônica (TIMÓTEO MVF, et al., 2020). Acredita-se também que, outro fator que pode favorecer a cronificação da hepatite é a maior ocorrência da infecção em pessoas em situação de pobreza e vulnerabilidade social, que, em geral, têm acesso restrito aos serviços públicos de saúde (MARQUES JVS, et al.,2019). Para Gomes RSL (2017), o risco de evoluir para um quadro crônico da doença é maior quando o indivíduo é infectado na infância, devido a um sistema imunológico prematuro.

A fim de evitar a evolução para forma crônica da doença, faz-se necessário além de um diagnóstico precoce $\mathrm{o}$ acesso ao tratamento adequado pois o acesso a um tratamento no estágio inicial da doença hepática é o ponto crucial para a prevenção de doenças hepatocelulares. Entretanto, segundo um relatório da OMS de 2018, a maior parte dos 71 milhões pessoas que têm infecção pelo vírus $C$ não recebe nenhum tratamento. Entretando, em alguns países nota-se um acesso maior ao tratamento com antivirais de ação direta (DAAs) e o Brasil está entre eles, junto com Egito, Geórgia, Austália, Ruanda e Espanha (DAS M, 2021).

A cronificação do HBV hoje, em outras palavras significa que o paciente terá essa doença por toda a vida (YOSHIDA O, et al., 2020; MAHATB MA, et al., 2018). Contudo um estudo, apresentado na American Association for the Study of Liver Diseases (AASLD) Liver Meeting em Boston, acompanhou 71 indivíduos infectados pelos vírus $\mathrm{B}$, durante 18 meses para avaliar a eficácia de uma vacina terapêutica contra HBV (NASVAC). Essa vacina é administrada por via nasal para tratar pacientes infectados, e tem como alvo dois antígenos diferentes do vírus da hepatite $B$ e os pesquisadores concluíram que a vacina é segura e eficaz para alcançar a cura funcional em pacientes com hepatite B crônica (YOSHIDA O, et al., 2020; MAHATB MA, et al., 2018). Uma possível esperança para cura.

\section{CONCLUSÃO}

Na capital alagoana as hepatites mais predominantes são B e C, onde a B foi a mais prevalente de 2010 a 2020. Sendo o sexo masculino, indivíduos na faixa etária dos 40 aos 59 anos, de raça parda, com ensino fundamental incompleto e em fase crônica da doença os mais acometidos. Sendo assim, em se tratando de doenças onde normalmente o diagnóstico é feito de forma tardia, a descrição do perfil epidemiológico é extremamente importante para controlar os fatores de risco. Assim como, é de suma importância verificar a cobertura vacinal da população para o vírus $B$, visto que a vacinação é o método mais eficaz para prevenir sua transmissão.

\section{REFERÊNCIAS}

1- ARAÚJO IN, et al. Perfil epidemiológico das hepatites B e C no estado do rio grande do norte. Revista Ciência Plural, 2020; 6(3): 35-52.

2- BARBOSA GFD, et al. Situação epidemiológica das hepatites virais no estado de goiás: 2013 a 2017. Revista científica da escola estadual de saúde pública de goiás "cândido santiago", 2019; 5(1):3-11.

3- BRASIL. Lei no 13.802, 2019. Disponível em: http://www.planalto.gov.br/ccivil_03/_ato20192022/2019/Lei/L13802.htm. Acessado em: 26 de junho de 2020. 
4- DAS M. Partnership to tackle HCV in low-income and Middle-income countries. The Lancet Oncology, 2021; 22(9):1218.

5- EVANGELISTA CBA, et al. Aspectos epidemiológicos da hepatite B no município de Teresina. Revista Eletrônica Acervo saúde, 2021; 13(5): 1-8.

6- FERREIRA VL, et al. Revisão sistemática da eficácia e da segurança das terapias livres de interferon para hepatite $\mathrm{C}$ crônica em pacientes coinfectados com o vírus da imunodeficiência humana. Revista saúde em debate, 2017; 41(115): 1212-1223.

7- GOMES AM, et al. Hepatites virais: uma análise clínico epidemiológica no estado do Tocantins nos últimos 18 anos. Revista de Patologia do Tacantins,2020; 7(2):107-113.

8- GOMES SRL. Caracterização dos genótipos e subgenótipos do vírus da hepatite bem portadores crônicos do Estado de Rondônia. Universidade Federal de Rondônia, 2017.

9- GUSMÃO BM, et al. Análise do perfil sociodemográfico de notificados para hepatite B e imunização contra a doença. J. res.: fundam. care. Online, 2017; 9(3): 627-633.

10-INTERFARMA. Associação da Indústria Farmacêutica de Pesquisa. Como as vacinas mudaram um país. São Paulo: Ativaonline Editora, 2016.

11-LANINI S, et al. Viral Hepatitis: Etiology, Epidemiology, Transmission, Diagnostics, Treatment, and Prevention. Infectious Disease Clinics of North America, 2019; 33(4): 1045-1062.

12-LEÃO DV, et al. Perfil epidemiológico das hepatites infectocontagiosas no estado do Pará. Revista Eletrônica Acervo Saúde, 2021;13 (4):1-11.

13-LEITE JMS, et al. Caracterização sociodemográfica e clínica de pacientes portadores de hepatite C crônica. Enfermeira Global, 2019; 55: 170-182.

14-MARQUES JVS, et al. Análise sociodemográfica das hepatites virais no estado do ceará. SANARE-Revista de Políticas Públicas, 2019; 18(2).

15-MAHTAB MAI, et al. Treatment of chronic hepatitis B naïve patients with a therapeutic vaccine containing HBs and $\mathrm{HBC}$ antigens (a randomized, open and treatment controlled phase III clinical trial). Plos one, 2018; 13 (18).

16-MINSTÉRIO DA SAÚDE. Boletim Epidemiológico de Hepatites Virais. Secretaria de vigilância em saúde. Departamento de doenças e condições crônicas e infecções sexualmente transmissíveis. 2020, 2020. Disponível em: http://www.aids.gov.br/pt-br/pub/2020/boletim-epidemiologico-hepatites-virais-2020. Acesso em: 20 de maio de 2021.

17-MINSTÉRIO DA SAÚDE. Boletim epidemiológico: hepatites virais 2019.Disponivel em: http://www.aids.gov.br/system/tdf/pub/2016/66453/boletim_hepatites_2019_c_.pdf?file=1\&t ype $=$ node\&id=66453\&force $=1>$. Acesso em: 20 de maio 2021.

18-MINSTÉRIO DA SAÚDE. Boletim epidemiológico: hepatites virais 2021.Disponível em: http://www.aids.gov.br/ptbr/pub/2021/boletim-epidemiologico-hepatites-virais-2021. Acesso em: 17 de janeiro de 2021.

19-MINSTÉRIO DA SAÚDE. Departamento de Vigilância das Doenças Transmissíveis. Secretaria de vigilância em saúde. Protocolo clínico e diretrizes terapêuticas para hepatite C e coinfecções, 2017. Disponível em: http://www.aids.gov.br/pt-br/pub/2017/protocolo-clinico-e-diretrizes-terapeuticas-para-hepatite-c-e-coinfeccoes. Acesso em: 11 de outubro de 2021

20-MINSTÉRIO DA SAÚDE. Manual técnico para o diagnóstico das hepatites virais. Secretaria de vigilância em saúde. Departamento de IST, AIDS e hepatites virais, 2018. Disponível em:http://www.aids.gov.br/ptbr/pub/2015/manualtecnico-para-o-diagnostico-das-hepatites-virais. Acesso em: 10 de maio de 2021.

21-NUNES F S, et al. Número de casos confirmados de hepatites virais no Brasil entre os anos de 2010 a 2015 . Revista Científica Multidisciplinar Núcleo do Conhecimento, 2020; 11(25): 71-80.

22-NUNES HM, et al., 2017. As hepatites virais: aspectos epidemiológicos, clínicos e de prevenção em municípios da Microrregião de Parauaupebas, Sudeste do estado do Pará, Brasil. Revista Pan-Amaz saúde, 2017; 8(2): 31 -37.

23-OLIVEIRA MS, et al. Hepatitis B and DELTA: clinical aspects of patients in tha Brazilian Western Amazonia. Revista Brasileira de Enfermagem,2019; 72(5); 1265-1270.

24-OLIVEIRA TJB, et al. Perfil epidemiológico dos casos de hepatite $C$ em um hospital de referência e infectocontagiosas no estado de Goiás. Revista Pan-Amazônica de Saúde, 2018; 9(1): 51-57.

25-PEREIRA VRZB, et al. Rick factors for hepatites B transmission inSouth Brasil. Memorias do Instituto Oswaldo Cruz, 2017; $112(8): 544-550$.

26-SANTOS ECC, MEMORIAN MAP. Situação epidemiológica brasileira sobre as hepatites B e C no período de 2000 a 2016. Brazilian Journal of Health Review, 2021; 4(5): 18612-18629.

27-SOTERO AS, et al. Perfil das hepatites virais em um estado nordeste brasileiro. Revista ciência e Saberes, 2020; 5(1).

28-SILVA TGQ, et al. Atualização em hepatite B: revisão bibliográfica. Brazillian Journal of Development, 2020: 6(12): 97930-97946.

29-STOCKDALE AJ, et al. The global prevalence of hepatite D vírus infection: systematic review and meta-analysis. Journal of Hepatology, 2020; 73(3): 523-532.

30-TIMÓTEO MVF, et al. Perfil epidemiológico das hepatites virais no Brasil. Research, Society ando development, 2020; 9(6).

31-UDOMPAP P, et al. Increasing prevalence of cirrhosis among U.S. adults aware or unaware of their chronic hepatitis $C$ virus infection. Journal of Hepatology, 2016; 64(5):1027-1032.

32-VIANA DR, et al. Hepatite B e C: diagnóstico e tratmento. Revista de Patologia do Tocantins, 2017; 4(3): 73-79.

33-YOSHIDA O, et al. HBsag reduction by nasal administration of a therapeutic vaccine containing hbsag and HBcag (nasvac) in patients with chronic HBV infection: the results of 18 months follow up. AASLS Livre Meeting Digital Experirence, 2020. 